INRA Prod. Anim.,

2013, 26 (4), 317-326

\title{
Amélioration génétique et utilisation des aliments à base de végétaux en pisciculture
}

\author{
R. LE BOUCHER ${ }^{1,2,3}$, M. DUPONT-NIVET ${ }^{1}$, S. LAUREAU ${ }^{4}$, L. LABBE' ${ }^{5}$, I. GEURDEN ${ }^{6}$ \\ F. MÉDALE ${ }^{6}$, B. CHATAIN ${ }^{2}$, M. VANDEPUTTE ${ }^{1,2}$, E. QUILLET \\ ${ }^{1}$ INRA, UMR1313 GABI, F-78350 Jouy-en-Josas, France \\ 2 Ifremer, UMR110 Intrepid, F-34250 Palavas-les-Flots, France \\ 3 AgroParisTech, UMR1313 GABI, F-75231 Paris 05, France \\ ${ }^{4}$ Aquanord, Ecloserie Marine de Gravelines, F-59820 Gravelines, France \\ 5 INRA, UE937 PEIMA, F-29450 Sizun, France \\ ${ }^{6}$ INRA, UMR1067 NuMeA, F-64310 St Pée-sur-Nivelle, France \\ Courriel : Edwige.Quillet@jouy.inra.fr
}

La production de poissons d'élevage est en constante augmentation, mais les composants traditionnels des aliments piscicoles, les huiles et les farines de poisson issues de la pêche, ne sont plus disponibles en quantité suffisante. Ils sont progressivement remplacés par des matières premières d'origine végétale. Des espèces essentiellement carnivores comme la truite ou le bar font preuve de capacités d'adaptation insoupçonnées à ces nouveaux ingrédients.

La consommation mondiale de poissons (pêche et pisciculture confondues) ne cesse d'augmenter. Depuis que les captures issues de la pêche ont atteint un plateau dans les années 1980, l'accroissement de la consommation de poisson repose exclusivement sur la croissance de la production piscicole (Tacon et Metian 2008), qui a ainsi connu depuis 20 ans une progression record en comparaison des filières de productions animales terrestres. Avec l'élevage des crustacés, la pisciculture est ainsi une des productions aquacoles à très forte croissance (taux de croissance sur les deux dernières décennies sept fois plus élevé que celui de la production de mollusques, FAO 2012). Encore confidentielle au début des années 1960, la contribution de la pisciculture à l'offre mondiale de poissons de consommation (40 Mt de production totale en eau douce, saumâtre et marine, FAO 2012) est désormais du même ordre de grandeur que celle de la pêche (de l'ordre de $60 \mathrm{Mt}$ sur la dernière décennie) et devrait continuer à augmenter.

La plupart des espèces de poissons d'élevage sont nourries avec des aliments composés, distribués en appoint (un tiers de la ration des poissons de bas niveau trophique) ou comme source exclusive de nourriture $(100 \%$ de la ration des poissons de haut niveau trophique). En pisciculture intensive, les aliments utilisés sont traditionnellement composés d'huiles et de farines (source protéique) de poisson. Ces deux ingrédients sont issus de la pêche dite minotière, laquelle exploite des stocks de petites espèces pélagiques, le plus souvent non directement consommées par 1'Homme. Mais ces stocks pélagiques étant pleinement exploités (Tacon et Metian 2009), des quotas ont été mis en place pour les protéger, et les quantités totales d'huiles et de farines disponibles plafonnent (environ $6 \mathrm{Mt}$ de farine et $1 \mathrm{Mt}$ d'huiles, Turchini et al 2009). L'utilisation de matières premières alternatives pour fabriquer les aliments piscicoles est incontournable pour répondre à l'augmentation des besoins. Les espèces de haut niveau trophique (salmonidés, poissons marins) sont particulièrement concernées, car leurs besoins élevés en protéines et en lipides en font de grandes consommatrices d'huiles et de farines de poisson (Médale et al 2013, ce numéro). La principale solution mise en œuvre a été une substitution croissante des huiles et des farines de poisson par des produits d'origine végétale (Naylor et al 2009), en particulier en Europe où la plupart des co-produits animaux ont été interdits depuis 2000 suite à la crise de la vache folle. Ainsi, entre 1997 et 2005, la proportion d'huile de poisson dans les aliments destinés aux espèces piscicoles marines a été diminuée de moitié (de 15 à 7\%) et la proportion de farine de poisson est passée de 50 à 30\% (Naylor et al 2009). La même tendance est observée dans les aliments utilisés en salmoniculture, l'incorporation de produits marins dans l'aliment étant passée de 45 à $24 \%$ entre 1995 et 2007 (Naylor et al 2000, 2009).

Cette évolution de la composition des aliments s'est appuyée sur les résultats de nombreux travaux réalisés en nutrition des poissons. Le choix des ingrédients et la formulation des aliments ont été affinés à partir des réponses des différentes espèces aux différents substituts végétaux (Médale et al 2013, ce numéro) et l'adaptation des procédés technologiques a permis d'améliorer les qualités nutritionnelles des composés végétaux. Toutefois, les taux de substitution très élevés restent associés à une dégradation de la croissance et dans certains cas, de la survie. L'origine de ces réductions de performances moyennes reste mal comprise, mais on observe au sein des lots nourris avec ces aliments substitués une importante variabilité des performances individuelles, certains individus s'adaptant mieux que d'autres. D'où l'idée qu'il pourrait exister au sein des populations d'élevage une variabilité génétique à l'origine de ces écarts, variabilité susceptible d'être exploitée par sélection pour accélérer l'adaptation des cheptels à des environnements alimentaires plus contraignants. 


\section{1 / Amélioration génétique et substitution des huiles et farines de poisson par des produits d'origine végétale : quelles sont les questions ?}

L'amélioration génétique des animaux domestiques par sélection est une pratique ancestrale. Les premiers agriculteurs/ éleveurs ont exploité la variabilité génétique des espèces pour les domestiquer et créer les races animales actuelles. D'abord fondée sur les seules observations de l'éleveur, la sélection s'est organisée dans le courant du $\mathrm{XX}^{\text {ème }}$ siècle grâce à la généralisation des principes de la génétique quantitative et de l'indexation des reproducteurs. L'élevage des poissons étant une activité relativement jeune (à l'exception notable de l'élevage de la carpe commune, domestiquée depuis plusieurs siècles), l'amélioration génétique des poissons s'est développée plus récemment, en même temps que se mettaient en place des élevages intensifs. Le premier schéma de sélection d'envergure a démarré en Norvège sur le saumon atlantique dans les années 1970 (Vandeputte et al 2009). Depuis, les schémas de sélection se sont multipliés dans le monde et concernent un nombre croissant d'espèces

Encadré. Héritabilité, intensité de sélection et progrès génétique.

L'héritabilité $\left(h^{2}\right)$ mesure, dans un environnement donné, la part de la variance d'un caractère déterminée par les gènes transmis par les parents. L'héritabilité varie de 0 (absence de variabilité génétique du caractère) à 1 (toute la variabilité des performances observées est d'origine génétique). L'héritabilité d'un caractère peut être estimée en étudiant la covariance des performances de groupes d'apparentés (souvent des familles de frères-sœurs dans le cas des poissons). On peut aussi estimer l'héritabilité réalisée à partir du progrès génétique effectivement obtenu sous l'effet de la sélection.

La pression de sélection désigne la proportion de reproducteurs utilisés pour produire la génération suivante. L'intensité de sélection i dépend directement de la pression de sélection (par exemple, l'intensité de sélection sera de 1,75 et 2,03 pour des pressions de sélection de 10 ou $5 \%$ respectivement).

Dans le cas simple d'une sélection individuelle (sélection dans laquelle les individus sont choisis à partir de leur performance propre), si $\mathrm{P}$ est la moyenne du caractère dans la population initiale et oP est l'écart-type phénotypique de ce caractère dans la population, le progrès génétique $\Delta \mathrm{P}$ (écart entre la performance moyenne des parents et la performance moyenne des descendants) attendu à chaque génération sera

$$
\Delta \mathrm{P}=\mathrm{i} \times \mathrm{h}^{2} \times \mathrm{OP}
$$

marines et d'eau douce et l'on dénombre aujourd'hui une centaine de programmes de sélection (saumons, truite, tilapia, bar, daurade, turbot, crevettes, mollusques, carpes...) (Gjedrem et al 2012).

Les objectifs de sélection de ces programmes d'amélioration génétique sont en premier lieu la croissance puis la qualité des produits (taux de lipides corporels, rendements en carcasse ou filet notamment), ainsi que les caractères de résistance aux maladies et d'adaptation (Vandeputte et al 2009). L'héritabilité d'un caractère et l'intensité de sélection exercée pour ce caractère déterminent le progrès génétique attendu à chaque génération (encadré). Pour la croissance, les héritabilités estimées sont en général relativement élevées $(0,3$ à 0,6$)$ chez les espèces piscicoles. Comme la fertilité des poissons est généralement

Tableau 1. Héritabilité des caractères de croissance chez différentes espèces de poissons nourries avec des aliments contenant des matières premières d'origine végétale.

\begin{tabular}{|c|c|c|c|c|c|c|c|c|}
\hline \multirow{2}{*}{ Espèce } & \multicolumn{2}{|c|}{$\begin{array}{c}\text { Taux de } \\
\text { substitution }^{(a)}\end{array}$} & \multirow{2}{*}{$\begin{array}{l}\text { Période } \\
\text { de suivi }\end{array}$} & \multirow{2}{*}{$\begin{array}{l}\text { Caractère } \\
\text { étudié }\end{array}$} & \multirow{2}{*}{$\begin{array}{l}\text { Performance } \\
\text { relative }^{(b)}\end{array}$} & \multicolumn{2}{|c|}{ Héritabilité } & \multirow{2}{*}{ Références } \\
\hline & $\mathrm{Hr} \%$ & Fr\% & & & & $\begin{array}{l}\text { Aliment } \\
\text { témoin }\end{array}$ & $\begin{array}{l}\text { Aliment } \\
\text { substitué }\end{array}$ & \\
\hline \multirow{6}{*}{$\begin{array}{l}\text { Truite } \\
\text { arc-en-ciel }\end{array}$} & 62,7 & 93,7 & $\begin{array}{l}1^{\text {er }} \text { repas- } \\
600 \mathrm{~g}\end{array}$ & Poids & $91 \%$ & $0,32 \pm 0,07$ & $0,31 \pm 0,07$ & $\begin{array}{c}\text { Pierce } \\
\text { et al (2008) }\end{array}$ \\
\hline & \multirow{5}{*}{100} & \multirow{5}{*}{100} & \multirow{5}{*}{$\begin{array}{l}1^{\text {er }} \text { repas- } \\
400 \mathrm{~g}\end{array}$} & Poids & $49 \%$ & $0,61 \pm 0,12$ & $0,67 \pm 0,12$ & \multirow{5}{*}{$\begin{array}{l}\text { Le Boucher } \\
\text { et al (2011b) }\end{array}$} \\
\hline & & & & Longueur & $81 \%$ & $0,55 \pm 0,11$ & $0,59 \pm 0,12$ & \\
\hline & & & & $\begin{array}{l}\text { Taux de croissance } \\
\text { journalier }\end{array}$ & $75 \%$ & $0,46 \pm 0,10$ & $0,65 \pm 0,12$ & \\
\hline & & & & $\begin{array}{l}\text { Rendement carcasse } \\
(\%)\end{array}$ & $92 \%$ & $0,48 \pm 0,11$ & $0,49 \pm 0,11$ & \\
\hline & & & & Rendement filets (\%) & ns & $0,21 \pm 0,18$ & $0,23 \pm 0,07$ & \\
\hline \multirow{4}{*}{ Corégone } & \multirow{4}{*}{0} & \multirow{4}{*}{67} & \multirow{4}{*}{$40 \mathrm{~g}-130 \mathrm{~g}$} & Gain journalier & $96 \%$ & $0,26 \pm 0,18$ & $0,20 \pm 0,15$ & \multirow{2}{*}{$\begin{array}{l}\text { Quinton } \\
\text { et al (2007a) }\end{array}$} \\
\hline & & & & Ingéré journalier & 1 & $0,23 \pm 0,15$ & $0,17 \pm 0,15$ & \\
\hline & & & & $\begin{array}{l}\text { Taux de lipides } \\
\text { (poisson entier) }\end{array}$ & I & $0,21 \pm 0,06$ & $0,18 \pm 0,06$ & Quinton \\
\hline & & & & $\begin{array}{l}\text { Taux de protéines } \\
\text { (poisson entier) }\end{array}$ & I & $0,07 \pm 0,04$ & $0,06 \pm 0,043$ & et al (2007b) \\
\hline \multirow{4}{*}{ Bar } & \multirow{4}{*}{100} & \multirow{4}{*}{100} & \multirow[b]{2}{*}{$190 \mathrm{~g}-650 \mathrm{~g}$} & Poids & $86 \%$ & $0,47 \pm 0,24$ & $0,36 \pm 0,18$ & \multirow{2}{*}{$\begin{array}{l}\text { Le Boucher } \\
\text { et al (2011a) }\end{array}$} \\
\hline & & & & $\begin{array}{l}\text { Teneur en lipides du } \\
\text { muscle }\end{array}$ & ns & $0,94 \pm 0,23$ & $0,56 \pm 0,22$ & \\
\hline & & & \multirow[b]{2}{*}{$2,5 \mathrm{~g}-110 \mathrm{~g}$} & Poids & $60 \%$ & $0,38 \pm 0,10$ & $0,18 \pm 0,07$ & \multirow{2}{*}{$\begin{array}{l}\text { Le Boucher } \\
\text { et al (2013) }\end{array}$} \\
\hline & & & & $\begin{array}{l}\text { Taux de croissance } \\
\text { journalier }\end{array}$ & ns & $0,33 \pm 0,10$ & $0,11 \pm 0,06$ & \\
\hline
\end{tabular}

(a) $\mathrm{Hr} \%, \mathrm{Fr} \%$ = taux de remplacement des huiles et des farines de poisson par des produits d'origine terrestre dans l'aliment expérimental $[\mathrm{Hr} \%=$ huile d'origine végétale terrestre $(\mathrm{g}) /$ huiles totales de l'aliment $(\mathrm{g}) ; \mathrm{Fr} \%=$ sources protéiques d'origine végétale $(\mathrm{g}) /$ sources protéiques totales de l'aliment $(\mathrm{g})$ ].

(b) Ecart relatif pour le caractère entre les lots ayant reçu l'aliment témoin et l'aliment substitué (= valeur moyenne du caractère avec aliment substitué/valeur moyenne avec aliment témoin en \%). 
élevée, il est possible d'appliquer de fortes pressions de sélection, la combinaison de ces deux facteurs permettant au final d'obtenir des progrès génétiques rapides, de l'ordre de 4 à $20 \%$ par génération pour la croissance (Olesen et al 2003, Chevassus et al 2004).

Dans ce contexte, une modification rapide de la composition des aliments piscicoles pose plusieurs questions :

- Y a-t-il des individus génétiquement mieux adaptés que d'autres à l'utilisation d'aliments à base de végétaux, et si oui, la variabilité génétique associée à ces différences de performance est-elle suffisamment importante et héritable pour envisager de sélectionner efficacement?

- Quel peut être l'impact d'un changement de régime alimentaire sur le progrès génétique obtenu par sélection ? Les individus sélectionnés avec les aliments actuels seraient-ils aussi les plus performants avec un aliment très fortement enrichi en ingrédients d'origine végétale ? Comment gérer efficacement les schémas de sélection pour adapter les cheptels aux évolutions futures du contexte alimentaire ?

- Enfin, s'il est confirmé que certains individus ont une aptitude spécifique à utiliser les aliments d'origine végétale, quelle est l'origine de cette adaptation et quels sont les mécanismes impliqués?

Pour répondre à ces questions jusque là peu explorées, les équipes de l'INRA et de l'Ifremer ont élaboré un programme de recherche (projet VEGEAQUA ${ }^{1}$ ) ciblé sur deux principales espèces produites en France, la truite arc-en-ciel (Oncorhynchus mykiss), une espèce d'eau douce, et le bar (Dicentrarchus labrax), une espèce marine, dont la production est encore fortement utilisatrice d'ingrédients issus des ressources marines. Une part importante des résultats présentés ici sont issus de ce programme.

\section{2 / Y a-t-il une variabilité génétique de la croissance avec un régime riche en pro- duits d'origine végétale ?}

Les premières démonstrations de l'existence d'une variabilité génétique pour l'utilisation d'aliments contenant des ingrédients végétaux chez les poissons d'élevage ont été obtenues chez la truite arc-en-ciel, en comparant les per- formances de familles nourries avec des aliments partiellement substitués (Blanc 2002, Dupont-Nivet et al 2009). Ces résultats qualitatifs ont été complétés par des estimations de l'héritabilité des principaux caractères de croissance d'animaux recevant, selon les expériences, des régimes partiellement ou totalement substitués (tableau 1). Dans ce tableau figurent notamment les résultats d'expérimentations réalisées dans le cadre du projet VEGEAQUA (Le Boucher et al 2011a, 2011b, 2013). La stratégie consiste à tester des régimes expérimentaux extrêmement contrastés, afin de faciliter l'identification de familles ou d'individus présentant des aptitudes spécifiques à l'utilisation de régimes pauvres en produits d'origine marine, voire, dans le cas du projet VEGEAQUA, totalement dépourvus de ces ingrédients (remplacement total des huiles et des farines de poisson). Le tableau 2 présente un exemple de composition d'aliments expérimentaux utili- sés chez la truite. L'aliment témoin est un aliment marin, qui ne contient pas de végétaux, hormis du blé qui sert principalement de liant. L'aliment végétal ne contient pas du tout de farine et huile de poisson. Les protéines sont apportées par un mélange de sources protéiques végétales, de façon à équilibrer le profil en acides aminés et réduire l'effet d'éventuels facteurs antinutritionnels. Pour couvrir les besoins, l'aliment est complémenté en certains acides aminés essentiels (la lysine notamment), en vitamines et en minéraux. Les lipides sont apportés par un mélange d'huiles végétales, dont l'huile de lin riche en acide linolénique C18:3 n-3, le précurseur de la synthèse d'acides gras polyinsaturés à longue chaîne (AGPI-LC) n-3 dont les poissons ont besoin pour leur survie et leur croissance (Médale et al 2013, ce numéro). Les deux aliments apportent des quantités d'énergie, de lipides et de protéines à peu près équivalentes.

Tableau 2. Exemple de composition d'un aliment totalement substitué (V) comparé à l'aliment marin $(M)$ utilisé chez la truite arc-en-ciel en début de croissance (d'après le Boucher et al 2011b, 2012).

\begin{tabular}{|c|c|c|}
\hline Ingrédients & M & V \\
\hline (g/kg) & & 0 \\
Farine de poisson & 692 & 250 \\
Gluten de maïs & 0 & 239 \\
Gluten de blé & 0 & 0 \\
Blé entier & 187 & 208 \\
Tourteau de soja & 0 & 70 \\
Lupin blanc & 0 & 0 \\
Pois, Aquatex & 0 & 0 \\
Huile de poisson & 81 & 62 \\
Huile de colza & 0 & 37 \\
Huile de lin & 0 & 24 \\
Huile de palme & 0 & 20 \\
Lecithine de soja & 0 & 15 \\
L-Lysine & 0 & 35 \\
CaHPO4.2H20 (18\%P) & 0 & 20 \\
Liant (alginate) & 20 & 10 \\
Minéraux Premix & 10 & 10 \\
Vitamines Premix & 10 & 23,2 \\
\hline Composition & $5,6,5$ & \\
Protéines (\% MS) & 15,6 & 0,5 \\
Lipides (\% MS) & & \\
Energie (kJ/g MS) & & 0,5 \\
\hline
\end{tabular}

MS : Matière Sèche

\footnotetext{
${ }^{1}$ Le projet VEGEAQUA (2009-2012) associait des acteurs du monde de la sélection aquacole (entreprises privées*, Syndicat des Sélectionneurs Avicoles et Aquacoles Français - SYSAAF) et de la recherche (INRA, Ifremer) sur la thématique « Génétique de l'adaptation aux aliments végétaux en aquaculture ». Il comportait des essais en stations expérimentales et des essais en fermes de production. Seuls les résultats des essais expérimentaux sont présentés ici. *Ecloserie Marine de Gravelines (porteur du projet), Ferme Marine du Douhet, Les Aquaculteurs Bretons, les Poissons du Soleil.
} 
Figure 1. Les différents types d'interaction entre génotype et environnement (comparaison des performances de 4 groupes génétiques dans les milieux 1 et 2).

A : absence d'interaction ; B : présence d'une interaction sans modification du classement entre les groupes ; C : présence d'une interaction avec modification du classement entre les groupes.

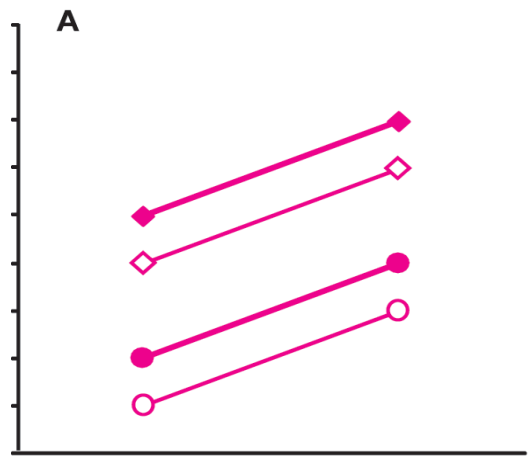

Milieu 1

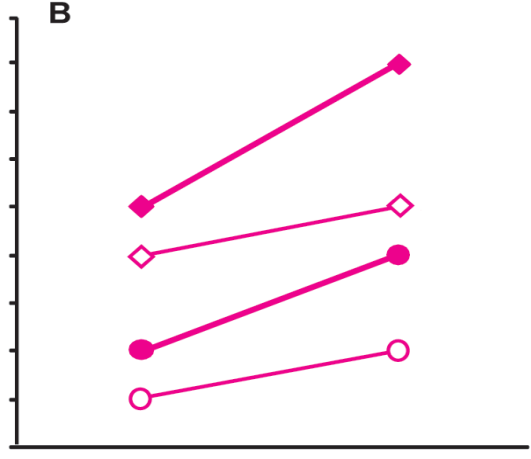

Milieu 1

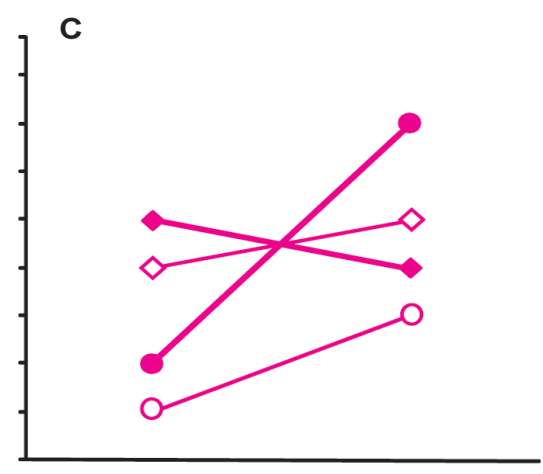

Milieu 1

Milieu 2

Tableau 3. Interactions génotype-aliment chez différentes espèces de poissons nourris avec des aliments contenant des huiles et des farines d'origine végétale.

\begin{tabular}{|c|c|c|c|c|c|c|c|}
\hline \multirow[t]{2}{*}{ Espèce } & \multicolumn{2}{|c|}{$\begin{array}{c}\text { Taux de } \\
\text { substitution }^{(a)}\end{array}$} & \multirow{2}{*}{$\begin{array}{l}\text { Période de } \\
\text { suivi }\end{array}$} & \multirow[t]{2}{*}{ Caractère étudié } & \multirow{2}{*}{$\begin{array}{l}\text { Corrélation } \\
\text { génétique }^{(b)}\end{array}$} & \multirow{2}{*}{$\begin{array}{l}\text { Interaction } \\
\text { génotype- } \\
\text { aliment }^{(\mathrm{c})}\end{array}$} & \multirow[t]{2}{*}{ Références } \\
\hline & $\mathrm{Hr} \%$ & $\mathrm{Fr} \%$ & & & & & \\
\hline \multirow{10}{*}{$\begin{array}{c}\text { Truite } \\
\text { arc-en-ciel }\end{array}$} & 0 & 47,6 & $1^{\text {er }}$ repas $-5 \mathrm{~g}$ & Poids & 1 & oui & Blanc (2002) \\
\hline & 0 & 87 & $\begin{array}{c}1^{\text {er }} \text { repas- } \\
380 \mathrm{~g}\end{array}$ & Poids, longueur & l & non & $\begin{array}{c}\text { Palti } \\
\text { et al (2006) }\end{array}$ \\
\hline & 62,7 & 93,7 & $\begin{array}{c}1^{\text {er }} \text { repas- } \\
600 \mathrm{~g}\end{array}$ & Poids & $0,73 \pm 0,13$ & oui & $\begin{array}{c}\text { Pierce } \\
\text { et al (2008) }\end{array}$ \\
\hline & \multirow{3}{*}{0} & \multirow{3}{*}{100} & \multirow{3}{*}{$1,2 \mathrm{~g}-4,2 \mathrm{~g}$} & Poids & 1 & oui & \multirow{3}{*}{$\begin{array}{l}\text { Dupont-Nivet } \\
\text { et al (2009) }\end{array}$} \\
\hline & & & & Ingéré & 1 & oui & \\
\hline & & & & $\begin{array}{l}\text { Efficacité } \\
\text { alimentaire }\end{array}$ & 1 & oui & \\
\hline & \multirow{4}{*}{100} & \multirow{4}{*}{100} & \multirow{4}{*}{$\begin{array}{c}1^{\text {er }} \text { repas- } \\
400 \mathrm{~g}\end{array}$} & Poids & $0,90 \pm 0,05$ & oui & \multirow{4}{*}{$\begin{array}{l}\text { Le Boucher } \\
\text { et al (2011b) }\end{array}$} \\
\hline & & & & Longueur & $0,90 \pm 0,05$ & oui & \\
\hline & & & & $\begin{array}{l}\text { Taux de croissance } \\
\text { journalier }\end{array}$ & $0,92 \pm 0,07$ & oui & \\
\hline & & & & $\begin{array}{l}\text { Rendement } \\
\text { carcasse }(\%)\end{array}$ & $0,90 \pm 0,06$ & oui & \\
\hline \multirow{5}{*}{ Corégone } & \multirow{5}{*}{0} & \multirow{5}{*}{67} & \multirow{5}{*}{$40 \mathrm{~g}-130 \mathrm{~g}$} & Gain journalier & $0,97 \pm 0,21$ & non & \multirow{3}{*}{$\begin{array}{l}\text { Quinton } \\
\text { et al (2007a) }\end{array}$} \\
\hline & & & & Ingéré & $0,93 \pm 0,28$ & non & \\
\hline & & & & Ingéré journalier & $1,00 \pm 0,95$ & non & \\
\hline & & & & $\begin{array}{l}\text { Taux de lipides } \\
\text { (poisson entier) }\end{array}$ & $0,95 \pm 0,15$ & non & Quinton \\
\hline & & & & $\begin{array}{l}\text { Taux de protéines } \\
\text { (poisson entier) }\end{array}$ & $0,89 \pm 0,53$ & non & et al (2007b) \\
\hline \multirow{4}{*}{ Bar } & \multirow{4}{*}{100} & \multirow{4}{*}{100} & \multirow[b]{2}{*}{$190 \mathrm{~g}-650 \mathrm{~g}$} & Poids & $0,51 \pm 0,34$ & oui & \multirow{2}{*}{$\begin{array}{l}\text { Le Boucher } \\
\text { et al (2011a) }\end{array}$} \\
\hline & & & & $\begin{array}{l}\text { Teneur en lipides } \\
\text { du muscle }\end{array}$ & $0,83 \pm 0,17$ & oui & \\
\hline & & & \multirow[b]{2}{*}{$2,5 \mathrm{~g}-110 \mathrm{~g}$} & Poids & $0,78 \pm 0,17$ & oui & \multirow{2}{*}{$\begin{array}{l}\text { Le Boucher } \\
\text { et al (2013) }\end{array}$} \\
\hline & & & & $\begin{array}{l}\text { Taux de croissance } \\
\text { journalier }\end{array}$ & $0,64 \pm 0,28$ & oui & \\
\hline $\begin{array}{l}\text { Tilapia } \\
\text { du Nil }\end{array}$ & I & I & 1 & Poids & l & oui & $\begin{array}{l}\text { Romana-Eguia } \\
\text { et Doyle (1992) }\end{array}$ \\
\hline
\end{tabular}

(a) $\mathrm{Hr} \%, \mathrm{Fr} \%$ = taux de remplacement des huiles et des farines de poisson par des produits d'origine terrestre dans l'aliment expérimental $[\mathrm{Hr} \%=$ huile d'origine végétale terrestre $(\mathrm{g}) /$ huiles totales de l'aliment $(\mathrm{g}) ; \mathrm{Fr} \%=$ sources protéiques d'origine végétale terrestre $(\mathrm{g})$ / sources protéiques totales de l'aliment $(\mathrm{g})$ ]

(b) Corrélation génétique entre les performances observées pour le caractère avec les régimes témoins et substitués. La corrélation peut varier de 0 à 1 . Des valeurs proches de 1 indiquent de faibles reclassements entre les groupes en fonction du régime.

(c) Interaction génotype-aliment. L'existence d'une interaction génotype-aliment est une autre indication de l'existence de réponses spécifiques de certaines familles à l'aliment substitué pour le caractère. 
Chez les salmonidés, l'utilisation d'aliments partiellement substitués a peu d'impact sur la survie. La substitution totale et concomitante des farines et huiles de poisson, testée chez la truite peut induire, de façon non systématique, une réduction modérée de la survie (Le Boucher et al 2011b, 2012). A l'inverse, chez les poissons marins, les taux de substitution élevés, en particulier pour les huiles, s'accompagnent de perturbations importantes (Médale et al 2013, ce numéro). Cependant, les données disponibles ne permettent pas de conclure sur l'existence d'une éventuelle variabilité entre groupes génétiques de la survie avec des régimes substitués.

Comme attendu, l'augmentation des taux de substitution s'accompagne d'une réduction marquée des performances de croissance (tableau 1). Par exemple, dans l'expérience de Le Boucher et al (2011b) les truites nourries pendant un an dès le premier repas exclusivement avec un aliment totalement dépourvu de produits d'origine marine sont deux fois plus petites que leurs soeurs nourries avec l'aliment « marin » (poids relatif $=49 \%$ ) alors que l'écart n'est que de quelques pourcents dans les études de Pierce $e t$ al (2008) ou Quinton et al (2007a) chez le corégone, espèce d'eau douce, en utilisant des régimes moins fortement substitués.

Malgré cette croissance réduite, on observe, dans la plupart des cas, des valeurs d'héritabilité moyennes à fortes avec l'aliment substitué (entre 0,11 et 0,67 ) pour la plupart des caractères étudiés, avec une tendance à l'accroissement des héritabilités pour l'aliment substitué par rapport à l'aliment témoin dans le cas de la truite arc-en-ciel et du corégone, et au contraire à la réduction dans le cas du bar, pour le taux de croissance journalier en particulier (tableau 1). On peut néanmoins conclure qu'il existe bien, chez les espèces étudiées, une variabilité génétique pour l'aptitude à la croissance à partir de régimes riches en produits végétaux, et que cette aptitude peut être sélectionnée efficacement.

\section{3 / Les interactions génotype- aliment : sélectionne-t-on les mêmes individus selon le régime?}

Les interactions génotype-environnement sont une préoccupation centrale en amélioration génétique, car elles conditionnent la manière dont le progrès génétique obtenu dans un environnement particulier sera conservé dans d'autres milieux. On distingue plusieurs situations, illustrées dans la figure 1 : les écarts entre les différents groupes génétiques et leur classement restent inchangés, quel que soit l'environnement (A); les écarts entre groupes sont modifiés quand l'environnement change, mais le classement reste identique (B) ; enfin, le classement, et éventuellement les écarts relatifs, entre groupes génétiques sont modifiés $(\mathrm{C})$, on parle alors d'interactions génotype-environnement. La corrélation génétique est une manière de quantifier l'importance des interactions. La corrélation génétique entre un caractère mesuré dans un environnement $\mathrm{X}$ et le même caractère mesuré dans l'environnement $Y$ est une indication de la similarité des caractères dans les deux environnements : plus la corrélation est forte, plus les caractères sont similaires dans les deux environnements, ce qui correspond à une interaction génotype-environnement faible. Si les reclassements sont importants (corrélations faibles), il devient nécessaire d'adapter le schéma de sélection pour prendre en compte les performances dans le milieu dans lequel seront élevés les descendants des candidats sélectionnés.

Le tableau 3 montre que des interactions génotype-aliment significatives sont fréquemment observées pour la croissance lorsque les taux de substitution des produits marins par des huiles et des farines végétales sont élevés. Malgré ces interactions, les corrélations génétiques restent dans l'ensemble assez fortes chez la truite ou le corégone (proches de 1), ce qui indique que le classement des différents groupes est assez peu modifié par le changement de régime. Les effets d'une substitution totale sont plus marqués chez le bar. Les interactions génotype-aliment semblent moins importantes pour les principaux caractères de qualité (teneur en lipides du muscle, rendement en carcasse) que pour les caractères de croissance.

Il convient cependant de rester prudent quant à l'impact potentiel de ces interactions, en particulier en raison de la faible précision de certaines estimations. En effet, une variation même modérée de la corrélation génétique peut avoir des répercussions importantes. Ainsi, avec une corrélation génétique de 0,7 , la valeur génétique estimée avec l'aliment témoin n'expliquera que la moitié de la variance génétique avec l'aliment substitué, et cette proportion diminuera très rapidement pour des valeurs de corrélation plus faibles. En cas d'interactions génotype-aliment, la précision, et donc l'efficacité de la sélection pratiquée avec le régime témoin risque de diminuer fortement si les générations suivantes sont nourries avec des aliments fortement substitués. L'évolution des corrélations génétiques dans le temps (figure 2) est un autre élément à prendre en compte pour organiser la sélection. Dans le cas du bar, les corrélations diminuent avec le temps, indiquant un renforcement progressif de l'effet différentiel des aliments. A l'inverse, chez la truite, la corrélation est faible en début d'essai et tend à augmenter par la suite. Ceci suggère un rôle déterminant des pha-

Figure 2. Evolution des corrélations génétiques pour le poids, pour des familles de truites ou de bars nourries avec un aliment contenant des huiles et farines de poisson et un aliment totalement substitué ne contenant que des huiles et des protéines d'origine végétale.

Truite arc-en-ciel : distribution de l'aliment substitué dès le premier repas (d'après Le Boucher et al 2011b). Bar - essai précoce : distribution de l'aliment substitué à partir de $2,5 \mathrm{~g}$ (d'après Le Boucher et al 2013). Bar - essai tardif : distribution de l'aliment substitué à partir de $192 \mathrm{~g}$ (d'après Le Boucher et al 2011a).

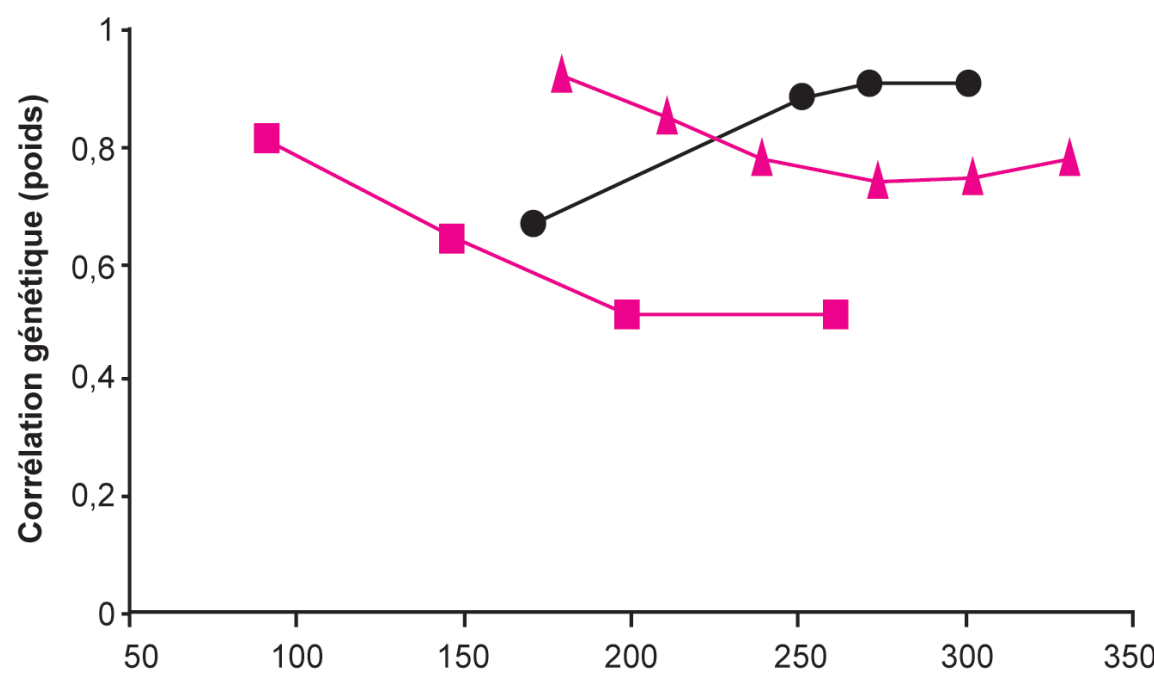

Truite arc-en-ciel $\neg$ Bar- essai précoce - Bar- essai tardif 
ses précoces pour l'adaptation à l'aliment substitué, avec un reclassement important des familles à ce stade. Un programme de sélection dont l'objectif serait d'obtenir des descendants adaptés aux différents aliments devrait donc s'appuyer sur des tris tardifs, ce qui peut constituer une contrainte par rapport à la conduite actuelle des schémas de sélection pour la croissance (application d'une sélection séquentielle par tris successifs, qui permet de réduire progressivement la biomasse dans les bassins d'élevage).

\section{4 / Et dans la pratique? Résultats de sélections expé- rimentales et évolution des schémas de sélection}

Les estimations de paramètres génétiques indiquent donc que la sélection pour la croissance avec des aliments contenant des matières premières végétales doit être efficace. Pour valider définitivement ces estimations, il reste à faire la démonstration qu'un progrès génétique peut effectivement être obtenu par la sélection.

\section{1 / Progrès réalisé par sélection expérimentale}

Deux expériences de sélection ont été conduites dans ce but chez la truite, l'une par une équipe américaine (Overturf et al 2012) et l'autre par l'INRA (Le Boucher et al 2012). Chez le bar, une expérience est en cours à l'Ifremer.

Dans l'expérience américaine réalisée chez la truite, un gain génétique très modeste (quelques pourcents) a été observé après quatre générations successives de sélection. Mais l'expérience souffre de l'absence de témoin non sélectionné permettant une mesure précise du gain obtenu (la population témoin a été « reconstituée » à partir des performances de plusieurs populations non sélectionnées). De plus, seule la farine de poisson était remplacée par des sources protéiques végétales, ce qui peut avoir limité l'impact de la substitution compte tenu du rôle primordial de la composition en huiles du régime. La sélection conduite par l'INRA a été réalisée avec un aliment totalement substitué (huiles et protéines d'origine exclusivement végétale). Les truitelles ont reçu cet aliment dès le premier repas, et ont été sélectionnées de manière séquentielle pour l'aptitude à survivre et grandir avec cet aliment jusqu'à l'âge de un an, de façon à ne conserver en fin de sélection que $3,25 \%$ des individus. Les animaux ainsi sélectionnés ont servi à produire la génération suivante (génération $\mathrm{S} 1$ ). Parallèlement, des animaux témoins (C1) ont été produits à partir de reproducteurs non sélectionnés provenant de la souche d'origine. Les performances des deux cohortes $\mathrm{S} 1$ et $\mathrm{C} 1$ ont été comparées en utilisant deux régimes alimentaires, un aliment marin contenant des huiles et des farines de poisson, et l'aliment totalement substitué utilisé pour la sélec- tion. Sans surprise, on observe que la croissance à un an est fortement diminuée avec l'aliment substitué (225 contre $526 \mathrm{~g}$ avec l'aliment marin dans la lignée témoin $\mathrm{C} 1$ ). En outre, quel que soit l'aliment, les descendants S1 ont une croissance supérieure à celle du lot $\mathrm{C} 1$ nourri avec le même aliment (figure 3).

Figure 3. Progrès génétique obtenu chez la truite arc-en-ciel pour le poids après une génération de sélection pour la croissance avec un aliment d'origine totalement végétale (d'après Le Boucher et al 2012).

Aliment $M$ : écart relatif entre la lignée sélectionnée $S 1$ et la lignée témoin $C 1$ nourries avec l'aliment marin. Aliment $\mathrm{V}$ : écart relatif entre ces deux mêmes lignées (S1, C1) lorsqu'elles sont nourries avec l'aliment substitué.

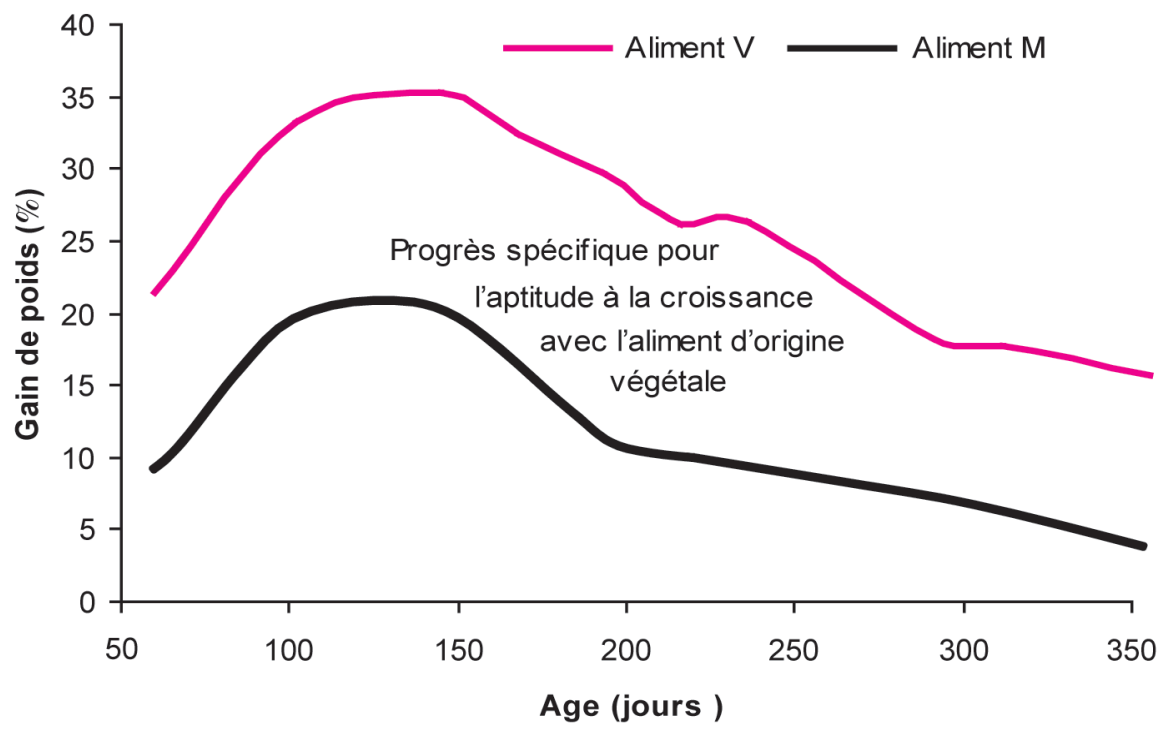

Figure 4. Effet de l'aliment et de la sélection sur l'homogénéité du poids chez la truite portion (d'après Le Boucher et al 2012).

La variabilité intra-population est mesurée avec le coefficient de variation du poids $(\mathrm{CV} \%$ = écart-type du caractère/moyenne du caractère, en \%). C1 et S1 correspondent au lot témoin et au lot sélectionné pendant une génération pour l'aptitude à grandir avec un aliment d'origine végétale. C1-AM et S1-AM correspondent au coefficient de variation du poids lorsque les deux lignées sont nourries avec l'aliment témoin contenant des huiles et farines de poisson. C1-AV et S1-AV correspondent aux coefficients de variation du poids lorsque les deux lignées sont nourries avec l'aliment substitué (contenant uniquement des huiles et des protéines d'origine végétale dans cette expérience).

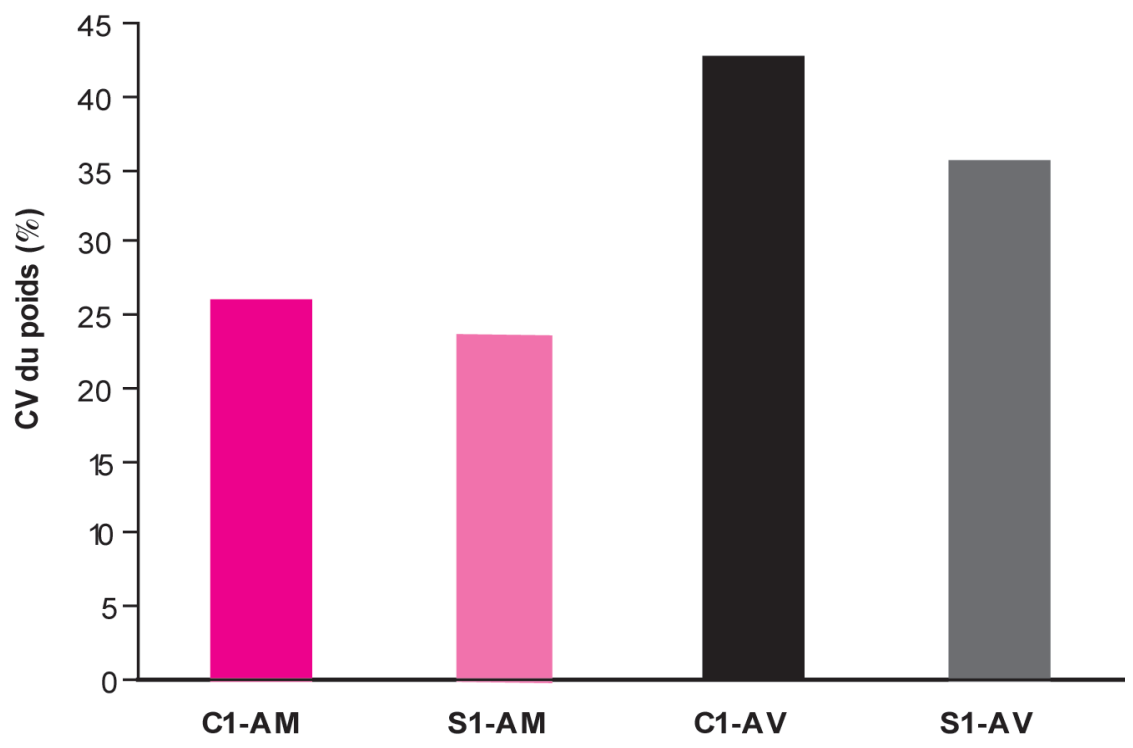


La sélection a donc augmenté le potentiel global de croissance. Cependant, conformément à ce que l'on pouvait attendre compte tenu des paramètres génétiques précédemment obtenus, la supériorité relative de la lignée sélectionnée est nettement plus marquée lorsque les animaux sont nourris avec l'aliment substitué. La différence de gain relatif entre les aliments correspond au progrès spécifique réalisé après une génération de sélection pour l'aptitude à la croissance avec l'aliment végétal, soit un progrès compris entre 10 et $20 \%$ sur la période d'étude. La sélection a également un effet positif sur la survie lorsque les lots sont nourris avec l'aliment végétal $(+21 \%$ par rapport à la survie du lot témoin $\mathrm{C} 1$ ), alors qu'aucun effet bénéfique de la sélection n'est observé dans les lots nourris avec l'aliment marin. L'essentiel des écarts de survie est établi dès les premières semaines d'alimentation, ce qui confirme l'importance des phases précoces dans l'adaptation au régime substitué. Enfin, on observe une forte augmentation de la variabilité des poids individuels au sein des lots lorsque ceux-ci sont nourris avec l'aliment substitué, le coefficient de variation moyen passant de 25 à $39 \%$ (figure 4). Toutefois, cette augmentation est beaucoup moins marquée dans le lot S1 que dans le lot témoin, ce qui constitue un avantage pour le lot sélectionné car l'homogénéité des performances facilite la gestion des cohortes en limitant le recours au tri. Par ailleurs, une variabilité phénotypique élevée est souvent révélatrice de problèmes d'élevage et/ou de qualité de l'environnement au sens large. L'amélioration conjointe de la croissance, de la survie et de l'homogénéité des poids suite à la sélection pour la croissance avec un aliment entièrement végétal montre donc un effet bénéfique de la sélection non seulement sur la croissance, mais plus globalement, sur l'adaptation des individus au nouveau contexte alimentaire.

Le progrès génétique réalisé à l'issue de cette sélection expérimentale a été comparé au progrès théorique attendu à partir des héritabilités estimées précédemment en utilisant le même aliment (Le Boucher et al 2011b). Si les réponses absolues à la sélection sont légèrement inférieures aux valeurs simulées (figure 5), le progrès génétique spécifique pour la croissance avec l'aliment substitué est conforme aux prédictions, ce qui conforte la validité des estimations des paramètres génétiques.

\section{2 / Scénarios de sélection et évo- lution des schémas de sélection}

Compte tenu du décalage entre la sélection et la diffusion du progrès génétique (2 à 4 ans minimum selon les espè- ces, et selon qu'il y a ou non une étape intermédiaire de multiplication), la conduite des programmes de sélection nécessite une forte capacité d'anticipation (évolution des conditions d'élevage des espèces, importance des différents caractères pour la commercialisation...). Dans le cas de la filière piscicole, la rapidité avec laquelle les formulations alimentaires sont susceptibles d'évoluer renforce l'intérêt d'une analyse prospective, susceptible d'aider les sélectionneurs à aménager leurs objectifs de sélection afin de fournir le produit le mieux adapté aux conditions d'élevage du moment. Ceci est d'autant plus important pour les sélectionneurs français qui ont une forte activité exportatrice d'œufs et d'alevins. En ce qui concerne l'évolution de la composition des aliments, la cohérence observée entre le progrès estimé et le progrès réalisé par sélection chez la truite permet de simuler avec une certaine confiance le progrès génétique attendu selon différents scénarios de sélection, et de proposer d'éventuelles évolutions des schémas de sélection.

Les simulations de progrès génétiques chez la truite et le bar ont été réalisées en utilisant les paramètres génétiques calculés avec les régimes expérimentaux du projet VEGEAQUA. Aussi, les hypothèses formulées pour ces estimations sont $i$ ) que seuls deux aliments de composition identique sont utilisés par les sélectionneurs et/ou les éleveurs ; ii)

Figure 5. Comparaison des réponses estimées et réalisées après une génération de sélection pour le poids lorsque les individus sélectionnés sont nourris avec un aliment d'origine entièrement végétale et lorsque les descendants sont nourris avec un aliment d'origine marine $(M)$ ou un aliment d'origine entièrement végétale (V).

La réponse estimée est calculée en simulant une sélection réalisée lorsque les individus atteignent un poids d'environ $200 \mathrm{~g}$, en utilisant les valeurs d'héritabilité et de corrélation génétique entre aliments estimées avec des individus de poids similaire dans l'expérience de Le Boucher et al (2011b). La réponse réalisée est celle obtenue après la sélection séquentielle entre 1 et $225 \mathrm{~g}$ décrite dans Le Boucher et al (2012).

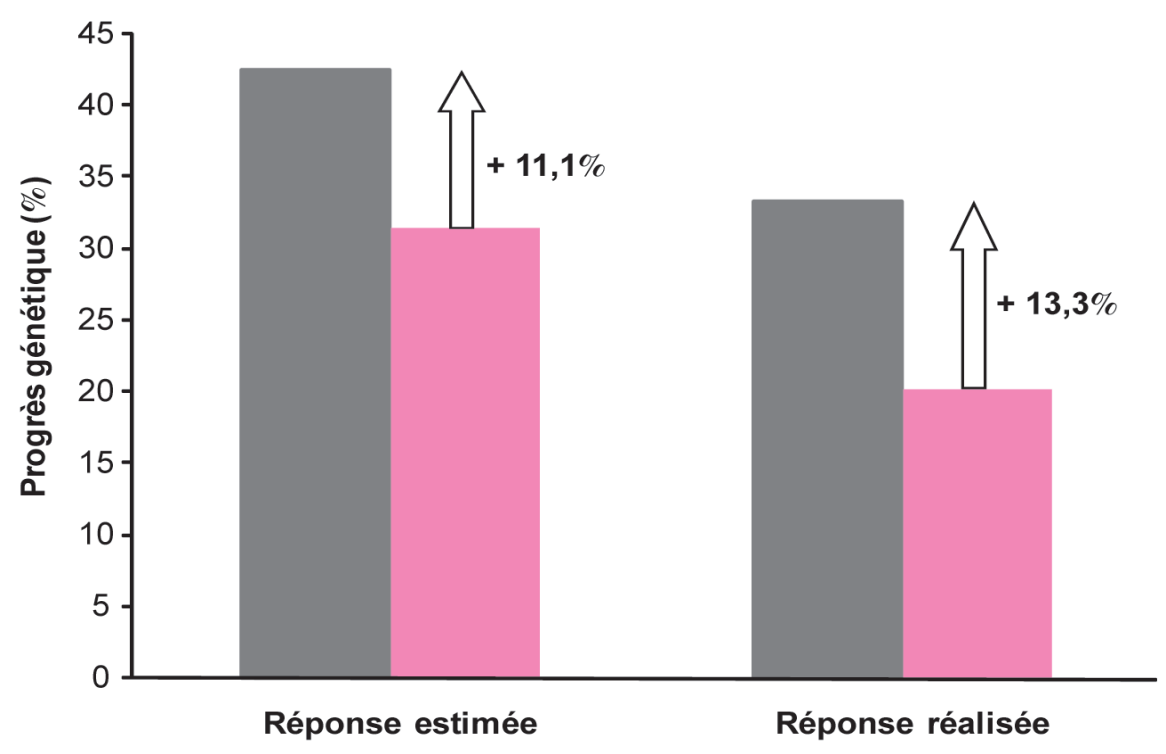

réponse avec aliment $\mathrm{V}$

que les populations sélectionnées présentent une variabilité génétique pour ment végétal similaire à celle observée chez la population expérimentale. Pour chaque espèce, quatre scénarios sont testés : la sélection des candidats est réalisée soit avec l'aliment substitué, soit avec l'aliment marin, les descendants (achetes par le client du sélectionneur) sont élevés avec l'un ou l'autre aliment. Tous les scénarios produisent du progrès génétique dans les deux espèces. Chez la truite, le progrès génétique maxinourrist obtenu lorsque les parents sont soit l'aliment avec lequel sont élevés les descendants $(+43 \%$ si les descendants sont aussi nourris avec l'aliment substitué, $+31 \%$ si les descendants sont nourris avec l'aliment marin). Les valeurs d'héritabilité légèrement plus élevées avec pensent la perte de précision de sélection liée aux interactions génotype-aliment. La situation est donc plutôt favorable, et chaque entreprise peut définir sa propre stratégie sans contrainte majeure. Chez le bar, il semble preférable de sélectionmême si les descendants sont élevés sur aliment d'origine végétale. Le faible progrès genetique attendu dans tous les ris avec l'aliment végétal résulte des valeurs assez modestes de l'héritabilité observées dans ce cas (voir tableau 1), 
Figure 6. Simulation du progrès génétique attendu après une génération de sélection pour le poids dans 4 scénarios d'organisation des schémas de sélection chez la truite arc-en-ciel et le bar.

Sélection $\mathrm{M}$ ou $\mathrm{V}$ : indique l'aliment utilisé, d'origine marine $(\mathrm{M})$ ou d'origine entièrement végétale $(\mathrm{V})$, pour sélectionner les reproducteurs. Production $\mathrm{M}$ ou $\mathrm{V}$ : indique l'aliment utilisé en production pour élever les descendants. Les valeurs des paramètres génétiques (héritabilités, corrélations génétiques) utilisées pour les simulations sont extraites des travaux de Le Boucher et al (2011b, 2013).

Truite arc-en-ciel

Bar

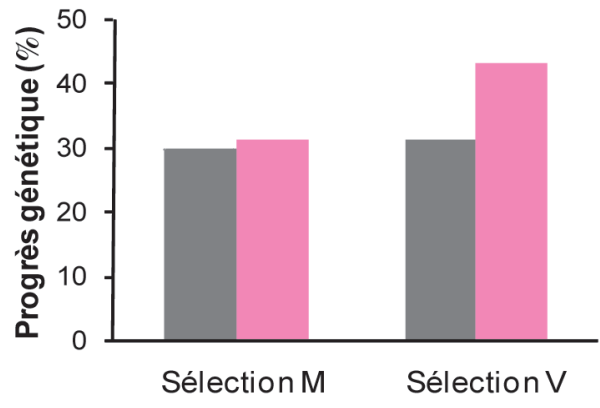

Production M

lesquelles pourraient être la conséquence de la distribution prolongée d'un régime totalement dépourvu de matières premières d'origine marine. En effet, les huiles végétales sont totalement dépourvues d'AGPI-LC n-3, et en particulier de l'acide eicosapentaénoïque (EPA) et de l'acide docosahexaénoïque (DHA) Or, ces deux acides gras sont indispensables chez les poissons marins (Montero et Izquierdo 2010), car dans ces espèces, leur néosynthèse à partir des précurseurs fournis dans l'aliment substitué (acide linolénique contenus dans l'huile de lin) est très réduite (Mourente et al 2005, Médale et al 2013, ce numéro). Il est probable que les bars nourris durablement avec un aliment exclusivement végétal n'ont pas réussi à couvrir leurs besoins en AGPI essentiels sur le long terme, ce qui a affecté leur état général comme en témoigne les mortalités observées au cours des essais et ne leur a pas permis d'exprimer pleinement leur potentiel de croissance. Il sera nécessaire, pour cette espèce, de tester des régimes moins fortement substitués pour consolider les conclusions.

\section{5 / Quels sont les mécanismes potentiels d'adaptation aux régimes substitués ?}

Il existe donc dans les populations d'élevage de poissons à haut niveau trophique comme la truite et le bar un réservoir de variabilité génétique permettant à certains individus de s'adapter aux aliments à base de matières premières d'origine végétale. Les mécanismes d'adaptation sous-jacents restent cependant à élucider, et peuvent se situer aux différentes étapes d'utilisation de l'aliment : ingestion et régulation de l'appétit, utilisation digestive et utilisation métabolique.

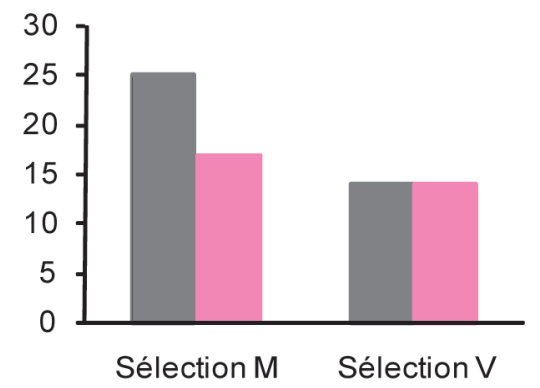

Production V

Certaines composantes importantes de la croissance ont déjà été explorées. La variabilité génétique pour l'ingéré et l'efficacité alimentaire a été démontrée avec les aliments conventionnels (Silverstein et al 2005, Kause et al 2006, Grima et al 2008, 2010a et b). Des études plus récentes, exploitant notamment des lignées isogéniques de truite, ont confirmé que cette variabilité existe également avec des aliments substitués, ainsi que des interactions génotypealiment (Dupont-Nivet et al 2009, Médale et al 2010). En revanche, la comparaison de la lignée de truite sélectionnée pendant une génération (S1) avec la lignée témoin $(\mathrm{C} 1)$ n'a pas permis de conclure définitivement sur le rôle de l'ingestion et de l'efficacité alimentaire dans la réponse à la sélection pour la croissance (Le Boucher et al 2011b, 2012). L'analyse des prochaines générations de sélection, une fois les différences entre lignées mieux établies, devrait permettre de valider ou non les tendances observées.

Au niveau métabolique, des différences génétiques et des interactions lignéealiment pouvant conduire à des reclassements marqués de certains génotypes ont également été mises en évidence pour les rétentions protéique, lipidique et énergétique (Médale et al 2010), ce qui confirme la complexité des mécanismes potentiellement mis en jeu dans la meilleure utilisation des aliments végétaux. Le lien potentiel entre ces caractères et l'adaptation aux aliments substitués reste à faire.

La capacité de synthèse et le métabolisme des AGPI-LC n-3 et n-6 est sans doute un des points critiques de l'adaptation des poissons aux régimes dépourvus d'huiles et de farines de poisson. Les AGPI ont un rôle important dans la fluidité des membranes cellulaires, au niveau cérébral notamment, et leur rôle sur la santé des poissons est bien établi (Montero et Izquierdo 2010). La teneur du muscle en AGPI (EPA et DHA notamment) est également un élément essentiel de la qualité diététique et nutritionnelle de la chair de poisson, principale source d'AGPI dans l'alimentation de l'Homme. Or, l'utilisation d'huiles végétales entraîne une diminution de la teneur en EPA et DHA de la chair, réduisant le bénéfice de la consommation de poisson (Torstensen et al 2005). La sélection d'animaux avec des teneurs accrues en AGPI n-3 dans la chair aurait donc un double intérêt. Des travaux récents ont montré des différences entre familles ou lignées pour la teneur en AGPI chez des salmonidés comme la truite (Médale et al 2010) et le saumon atlantique (Leaver et al 2011), ce qui suggère des différences génétiques de capacité de synthèse ou d'utilisation de certains AGPI. Chez le saumon, le pourcentage d'AGPI n-3 du muscle est fortement héritable $\left(\mathrm{h}^{2}=\right.$ $0,77 \pm 0,14$, Leaver et al 2011). De façon intéressante, la sélection expérimentale chez la truite semble avoir modifié le métabolisme lipidique global (en augmentant l'oxydation globale des lipides et en favorisant une utilisation différentielle de certains acides gras) et avoir légèrement amélioré la synthèse nette de DHA (Le Boucher 2011). Ce résultat conforterait l'hypothèse d'un rôle clé de la capacité de synthèse en AGPI essentiels dans l'adaptation aux aliments dépourvus de produits marins et ouvre des pistes intéressantes pour améliorer l'adaptation des cheptels aux régimes substitués.

Chez le bar en revanche, cette piste semble moins prometteuse. Les capacités de synthèse des AGPI n-3 à partir des précurseurs sont extrêmement réduites chez les espèces marines car certaines enzymes clé sont peu actives (D5 désaturase notamment, Geay et al 2010). Il faudra sans doute rechercher ailleurs l'origine des différences de potentiel de croissance observées dans cette espèce avec des régimes substitués.

\section{Conclusions et perspectives}

L'élevage et la consommation de poissons d'élevage en Europe concernent presque exclusivement des espèces de haut niveau trophique (truite, saumon, bar, daurade, turbot). On sait maintenant que plusieurs de ces espèces présentent une variabilité génétique sélectionnable leur permettant de s'adapter à des aliments particulièrement riches en matières premières (huiles et protéines) d'origine végétale. Ces résultats ont certes été obtenus avec des formules expérimentales optimisées, trop coûteuses pour une utilisation commerciale. Mais l'utilisation 
de ces formulations extrêmes a révélé la présence dans les espèces d'élevage d'un potentiel naturel d'adaptation insoupçonné. En pisciculture marine et d'eau douce, comme dans les autres productions animales, l'environnement alimentaire sera de plus en plus contraignant. L'incorporation de matières premières entrant moins en compétition avec l'alimentation humaine sera la prochaine transition à laquelle la filière doit se préparer. Les produits d'origine végétale resteront attractifs car disponibles en grandes quantités. Dans ce contexte, la possibilité de sélectionner pour la capacité à utiliser les ingrédients végétaux ouvre des perspectives très prometteuses pour la filière. Des travaux complémentaires se pour- suivent pour valider ces conclusions en conditions de production et pour identifier les mécanismes d'adaptation mis en jeu. La connaissance fine de ces mécanismes facilitera l'identification des verrous à l'origine des réductions de croissance et facilitera l'amélioration des formulations. Cela permettra aussi de repérer des marqueurs de l'adaptation (marqueurs génétiques ou physiologiques) utiles pour sélectionner plus efficacement. En exploitant de façon complémentaire programmes d'amélioration génétique et parcours alimentaires raisonnés, la filière doit pouvoir évoluer vers un mode de production plus durable, tout en continuant à fournir des produits de qualité au consommateur.

\section{Remerciements}

Les auteurs remercient le SYSAAF et les entreprises partenaires du projet VEGEAQUA. Ce projet a été labellisé par les pôles de compétitivité AQUIMER, Mer Bretagne et Mer PACA, et financé par le FUI (Fond Unique Interministériel), Oséo, les Régions Bretagne, Languedoc-Roussillon, Nord-Pas-de Calais et Poitou-Charentes, ainsi que l'Europe (fonds FEDER). Les génotypages permettant de reconstituer la généalogie des familles utilisées dans le projet ont été réalisés à LABOGENA.

\section{Références}

Blanc J.M., 2002. Interaction between diet and genetic aptitude for weight and growth in juvenile rainbow trout, Ocorhynchus mykiss (Walbaum). Aquac. Res., 33, 563-568.

Chevassus B., Quillet E., Krieg F., Hollebecq M.G., Mambrini M., Fauré A., Hiseux J.P., Vandeputte M., 2004. Enhanced individual selection for selecting fast growing fish: the 'PROSPER' method, with application on brown trout. Genet. Select. Evol., 36, 643661 .

Dupont-Nivet M., Médale F., Léonard J., Le Guillou S., Tiquet F., Quillet E., Geurden I., 2009. Evidence of genotype-diet interactions in the response of rainbow trout (Oncorhynchus mykiss) clones to a diet with or without fishmeal at early growth. Aquaculture, 295, 15-21.

FAO, 2012. La situation mondiale des pêches et de l'aquaculture 2012. FAO, Rome, $241 \mathrm{pp}$. (http://www.fao.org/docrep/016/ i2727f/i2727f00.htm).

Geay F., Santigosa I Culi E., Corporeau C., Boudry P., Dreano Y., Corcos L., Bodin N., Vandeputte M., Zambonino-Infante J.L., Mazurais D., Cahu C.L., 2010. Regulation of FADS2 expression and activity in European sea bass (Dicentrarchus labrax, L.) fed a vegetable diet. Comp. Biochem. Physiol., B, Biochem. Mol. Biol., 156, 237-243.

Gjedrem T., Robinson N., Rye M., 2012. The importance of selective breeding in aquaculture to meet future demans for animal protein: a review. Aquaculture, 350, 117-129.

Grima L., Quillet E., Boujard T., RobertGranié C., Chatain B., Mambrini M., 2008. Genetic variability in residual feed intake in rainbow trout clones and testing of indirect selection criteria. Genet. Select. Evol., 40, 607 624.

Grima L., Vandeputte M., Ruelle F., Vergnet A., Mambrini M., Chatain B., 2010a. In search for indirect criteria to improve residual feed intake in sea bass (Dicentrarchus labrax). Part I: phenotypic relationship between residual feed intake and body weight variations during feed deprivation and re-feeding periods. Aquaculture, 300, 50-58.

Grima L., Chatain B., Ruelle F., Vergnet A., Launay A., Mambrin, M., Vandeputte M., 2010 b. In search for indirect criteria to improve feed utilization efficiency in sea bass (Dicentrarchus labrax). Part II: Heritability of weight loss during feed deprivation and weight gain during re-feeding periods. Aquaculture, 302, 169-174.

Kause A., Tobin D., Houlihan D.F., Martin S.A.M., Mantysaari E.A., Ritola O., Ruohonen K., 2006. Feed efficiency of rainbow trout can be improved through selection: different genetic potential on alternative diets. J. Anim. Sci., 84, 807-817.

Leaver M.J., Taggart J.B., Villeneuve L., Bron E.J., Guy D.R., Bishop S.C., Houston R.D., Matika O., Tocher D.R., 2011. Heritability and mechanisms of n-3 long chain polyunsaturated fatty acid deposition in the flesh of Atlantic salmon. Comp. Bioch. Physiol. D : Genomics and Proteomics, 6, 62-69.

Le Boucher R., 2011. Génétique de l'utilisation de produits d'origine végétale chez la truite arc-en-ciel (Oncorhynchus mykiss) et le bar européen (Dicentrarchus labrax). Thèse de Docteur Ingénieur AgroParisTech, 282pp.

Le Boucher R., Vandeputte M., DupontNivet M., Quillet E., Mazurais D., Robin J., Vergnet A., Médale F., Kaushik S., Chatain B, 2011a. A first insight into genotype-diet interactions in European sea bass (Dicentrarchus labrax) in the context of plant-based diet use. Aquac. Res., 42, 583-592.

Le Boucher R., Quillet E., Vandeputte M., Lecalvez J.M., Goardon L., Chatain B., Médale F., Dupont-Nivet M., 2011b. Plant-based diet in rainbow trout (Oncorhynchus mykiss W.): are there genotype-diet interactions for main production traits when fish are fed marine vs plant-based diets from the meal? Aquaculture, 321, 41-48.

Le Boucher R., Dupont-Nivet M., Vandeputte M., Kerneïs T., L. Goardon L., Labbé L. Chatain B., Médale F., Quillet, E., 2012. Selection for adaptation to dietary shifts: towards sustainable breeding of carnivorous fish. PLoS ONE, 7, e44898.

Le Boucher R., Vandeputte M., DupontNivet M., Quillet E., Ruelle F., Vergnet A., Kaushik S., Allamellou J. M., Médale F., Chatain B., 2013. Genotype by diet interactions in European sea bass (Dicentrarchus labrax L.): Nutritional challenge with totally plant-based diets. J. Anim. Sci., 91, 44-56. doi:10.2527/ jas.2012-5311.

Médale F., Dupont-Nivet M., Rimoldi S. Yilmaz H.A., Terrier F., Corraze G., Quillet E., Geurden I., 2010. Responses of rainbow trout clonal lines to plant-based feed evidence genotype $\mathrm{x}$ diet interactions. Proc. $14^{\text {th }}$ Int. Symp. Fish Nutr. Feeding, Qingdao, China.

Médale F., Le Boucher R., Dupont-Nivet M., Quillet E., Aubin J., Panserat S., 2013. Des aliments à base de végétaux pour les poissons d'élevage. INRA Prod. Anim, 26, 303-316.

Montero D., Izquierdo M. S., 2010. Health and welfare of fish fed vegetable oils as alternative lipid sources to fish oil. In : Fish oil replacement and alternative lipid sources in aquaculture feeds. Turchini G.M., Ng W.K., Tocher D.R. (Eds). CRC Press, USA, p439.

Mourente G., Dick J.R., Bell J.G., Tocher D.R., 2005. Effect of partial substitution of dietary fish oil by vegetable oils on desaturation and [beta]-oxidation of [1-14C]18:3n-3 (LNA) and $[1-14 C] 20: 5 n-3$ (EPA) in hepatocytes and enterocytes of European sea bass (Dicentrarchus labrax L.). Aquaculture, 248, 173-186

Naylor R.L., Goldburg R.J., Primavera J.H., Kautsky N., Beveridge M.C.M., Clay J., Folke C., Lubchenco J., Mooney H., Troell M., 2000. Effect of aquaculture on world fish supplies. Nature, 405, 1017-1024.

Naylor R.L., Harry R.W., Bureau D., Chiu A., Elliott M., Farrell A.P., Forster I., Gatlin D.M., Goldburg R.J., Hua K., Nichols P.D., 2009. Feeding aquaculture in an era of finite resources. Proc. Natl. Acad. Sci. USA, 106, 15103-15110

Olesen I., Gjedrem T., Bentsen H. B., Gjerde,B., Rye M., 2003. Breeding programs for sustainable aquaculture. J. Appl. Aquac., 13, 179-204.

Overturf K, Barrosa F.T., Hardy R.W., 2012. Effect and interaction of rainbow trout strain (Oncorhynchus mykiss) and diet type on growth and nutrient retention. Aquac. Res., 44, 604611.

Palti Y., Silverstein J.T., Wieman H., Phillips J.G., Barrows F.T., Parsons J.E., 2006. Evaluation of family growth response to fish- 
meal and gluten-based diets in rainbow trout (Oncorhynchus mykiss). Aquaculture, 255, 548-556.

Pierce L.R., Palti Y., Silverstein J.T., Barrows F.T., Hallerman E.M., Parsons J.E., 2008. Family growth response to fishmeal and plant-based diets shows genotype $\mathrm{x}$ diet interaction in rainbow trout (Oncorhynchus mykiss). Aquaculture, 278, 37-42.

Quinton C.D., Kause A., Ruohonen K., Koskela J., 2007a. Genetic relationships of body composition and feed utilization traits in European whitefish (Coregonus lavaretus L.) and implications for selective breeding in fishmeal- and soybean meal-based diet environments. J. Anim. Sci., 85, 3198-3208.

Quinton C.D., Kause A., Koskela J., Ritola O., 2007b. Breeding salmonids for feed efficiency in current fishmeal and future plantbased diet environments. Gen. Sel. Evol., 39, 431-446.

Romana-Eguia M.R.R., Doyle R.W., 1992. Genotype-environment interaction in the response of three strains of Nile tilapia to poor nutrition. Aquaculture, 108, 1-12.

Silverstein J.T., Hostuttler M., Blemiings K.P., 2005. Strain differences in feed efficiency measured as residual feed intake in individually reared rainbow trout (Oncorhynchus mykiss) (Walbaum). Aquac. Res., 36, 704-711.

Tacon A.G.J., Metian M., 2008. Global overview on the use of fish meal and fish oil in industrially compounded aquafeeds: Trends and future prospects. Aquaculture 285, 146158
Tacon A.G.J., Metian M., 2009. Fishing for aquaculture: non-food use of small pelagic forage fish - a global perspective. Rev. Fish. Sci., 17, 305-317.

Torstensen B.E., Bell J.G., Rosenlund G., Henderson R.J., Graff I.E., Tocher D.R., Lie, O. Sargent J.R., 2005.Tailoring of Atlantic salmon (Salmo salar L.) flesh lipid composition and sensory quality by replacing fish oil with a vegetable oil blend. J. Agric. Food Chem., 53, 10166-10178.

Turchini G.M., Torstenten B.E., Ng W.K., 2009. Fish oil replacement in finfish nutrition. Reviews in Aquaculture, 1, 10-57.

Vandeputte, M., Baroiller, J.F., Haffray, P., Quillet, E., 2009. Amélioration génétique des poissons : quelles réalisations et quels défis pour demain? Cah. Agric., 18, 1-7.

\section{Résumé}

La production mondiale de poissons d'élevage augmente rapidement alors que les principaux ingrédients des aliments pour poissons (huiles et farines de poisson issues de la pêche) sont en quantité désormais insuffisante pour répondre à la demande. Cette situation, en Europe notamment, a conduit au remplacement progressif des produits marins par des matières premières végétales. Mais les taux de substitution élevés provoquent des réductions importantes des performances (croissance, voire survie) chez les espèces d'élevage de haut niveau trophique, à forts besoins en protéines et lipides. L'étude du potentiel d'adaptation de plusieurs de ces espèces à des aliments fortement ou totalement substitués (sans huiles ni farines de poisson) a révélé qu'il est possible de sélectionner au sein des populations d'élevage des individus à forte capacité de croissance avec des régimes à base de végétaux. Les mécanismes à l'origine de cette adaptation restent à identifier. Les analyses montrent aussi que les groupes les plus performants varient en fonction de l'aliment (marin ou substitué). Ces interactions génotype-aliment peuvent compliquer la gestion d'un programme de sélection si les reproducteurs sont sélectionnés avec un aliment différent de celui utilisé pour élever leurs descendants. Des simulations du progrès génétique attendu dans différents scénarios alimentaires permettent d'identifier quelques pistes pour optimiser la sélection chez la truite arcen-ciel et le bar, espèces majeures de la pisciculture d'eau douce et marine en France. La combinaison judicieuse de programmes d'amélioration génétique et de parcours alimentaires raisonnés pourrait aider la filière piscicole à évoluer vers un mode de production moins dépendant des ressources naturelles marines, tout en continuant à fournir des produits de qualité au consommateur.

\section{Abstract \\ Selective breeding and shift to plant-based diets in fish farming}

World fish farm production is growing rapidly. However, the supply of fishmeal and fish oil, the major ingredients of fish diets, has reached a limit because of the limits in the exploitation of wild fish stocks that cannot be increased to meet a growing demand. The main response, especially in Europe, has been the progressive replacement of fishmeal and fish oil by plant products. However, the strong reduction of growth and sometimes survival of fish fed highly substituted diets has urged research on the genetic potential for adaptation to plant-based diets. Recent results clearly demonstrate that fish can be selected for their capacity to grow when fed plantbased diets, even though the underlying mechanisms remain unknown. Genotype-diet interactions have been shown, indicating that the best performers differ according to the diet. Such interactions may make the management of breeding programmes difficult, as progeny may be reared with a diet differing from the one used to select the breeders. Simulations of the expected genetic gain according to different scenarios can help manage selection programmes for sea bass and rainbow trout, the main farmed species in France. Using both selective breeding and optimized diets provide the opportunity to increase sustainability of fish farming, whilst maintaining product quality.

LE BOUCHER R., DUPONT-NIVET M., LAUREAU S., LABBÉ L., GEURDEN I., MÉDALE F., CHATAIN B., VANDEPUTTE M., QUILLET E., 2013. Amélioration génétique et utilisation des aliments à base de végétaux en pisciculture. INRA Prod. Anim., 26, 4, 317-326. 